\title{
PARABOLICITY, THE DIVERGENCE THEOREM FOR $\delta$-SUBHARMONIC FUNCTIONS AND APPLICATIONS TO THE LIOUVILLE THEOREMS FOR HARMONIC MAPS
}

\author{
Atsushi Atsuji
}

(Received October 2, 2003, revised March 28, 2005)

\begin{abstract}
We show that the parabolicity of a manifold is equivalent to the validity of the 'divergence theorem' for some class of $\delta$-subharmonic functions. From this property we can show a certain Liouville property of harmonic maps on parabolic manifolds. Elementary stochastic calculus is used as a main tool.
\end{abstract}

0. Introduction. This note was inspired by the following two results concerning the Liouville property of harmonic maps and subharmonic functions of finite energy.

THEOREM 1 (Cheng-Tam-Wan [5]). Let $f: M \rightarrow N$ be a harmonic map of finite energy from a complete Riemannian manifold $M$ to a Cartan-Hadamard manifold $N$ (i.e. $N$ is a simply connected, complete Riemannian manifold of nonpositive sectional curvature). If $M$ does not admit nonconstant bounded harmonic functions, then $f$ is constant.

They actually showed that under the assumption on the source manifold, the image of the harmonic map should be bounded. The above result is obtained by combining this with a result due to Kendall [21] to the effect that if $M$ does not admit nonconstant bounded harmonic functions and $f$ is bounded, then $f$ is constant.

The second is a divergence theorem due to Takegoshi.

THEOREM 2 (Takegoshi [25]). Let $M$ be a complete Riemannian manifold and $u$ a $C^{2}$-function on $M$. Suppose that u satisfies

$$
|\nabla u|^{2}=o\left(\int_{1}^{r} \frac{1}{d V(t) / d t}\right), \quad \text { and } \quad(\Delta u)^{-} \in L^{1}(M),
$$

where $V(t)$ is the Riemannian volume of a geodesic ball of radius $t$. Then $(\Delta u)^{+} \in L^{1}(M)$ and

$$
\int_{M} \Delta u d v=0
$$

2000 Mathematics Subject Classification. Primary 31C05; Secondary 58J65.

Key words and phrases. Dirichlet form, Martingale, Harmonic map, Liouville theorem, $\delta$-subharmonic functions.

Partly supported by the Grant-in-Aid for Scientific Research (C), Japan Society for the Promotion of Science. 
He improved the result due to Cheng et al. in the case where

$$
\int_{1}^{\infty} \frac{1}{d V(t) / d t}=\infty .
$$

It is known that this condition on the volume growth implies the parabolicity of the manifolds (cf. [10, 25]). We say that a Riemannian manifold $M$ is parabolic or recurrent if $M$ does not admit any nonconstant bounded subharmonic function. This is equivalent to saying that no positive Green function exists on $M$, namely,

$$
g(x, y)=\int_{0}^{\infty} p(t, x, y) d t=\infty, \quad x, y \in M,
$$

where $p(t, x, y)$ is the heat kernel for $(1 / 2) \Delta_{M}$ where $\Delta_{M}$ is the Laplacian on $M$ with respect to the Riemannian metric. This implies that the Brownian motion on $M$ is recurrent. If the Brownian motion on $M$ is transient (i.e., nonparabolic), there is a general result about subharmonic functions of finite Dirichlet integral. Then Chang-Tam-Wan's result is easier in this case. (In the last section we give a proof of their result in transient case. The method used there is applicable in the more general cases.) Hence, in this paper we devote ourselves mainly to the parabolic case. To improve the above results, we use a probabilistic method including the ergodic theorems for recurrent Markov processes. This probabilistic method enables us to extend the above result to the case of $L$-harmonic maps.

We first show a variant of Takegoshi's result in the general setting. The usual divergence theorem implies that for any $\phi \in C_{0}^{\infty}(M)$

$$
\int_{M} \Delta \phi d v=0
$$

Thus, Takegoshi's result implies that some parabolicity allows a divergence theorem to hold for functions that do not necessarily have compact support. We show that the validity of such a divergence theorem for some class of $\delta$-subharmonic functions gives a characterization of the parabolicity of a manifold.

In the Riemannian case our results can be translated into the following. We consider $\Delta u$ in the sense of distribution. Then it can be regarded as a signed measure. $(\Delta u)^{+}$and $(\Delta u)^{-}$ denote the positive and negative parts of $\Delta u$, respectively, so that they are measures.

THEOREM 3. The following properties are equivalent.

(i) $M$ is a parabolic manifold.

(ii) If $u$ is a $\delta$-subharmonic function of finite Dirichlet integral, then

$$
\int_{M}(\Delta u)^{+}=\int_{M}(\Delta u)^{-} \quad(\leq \infty) .
$$

(iii) If $u$ is a bounded $\delta$-subharmonic function, then

$$
\int_{M}(\Delta u)^{+}=\int_{M}(\Delta u)^{-} \quad(\leq \infty) .
$$

This characterization in a general setting is given in Theorem 20 below. We would like to emphasize in this paper that this divergence theorem in principle joins stochastic calculus 
with elementary tools of analysis on manifolds such as the coarea formula and Green's formula. Actually we give a general version of the coarea formula in Proposition 9 and Green's formula in Theorem 25 for $\delta$-subharmonic functions. We also show in Proposition 31 that usual Green's formula can be derived from our divergence theorem by means of stochastic calculus.

As for an application to harmonic maps we have the following.

THEOREM 4. Let $M$ be a parabolic Riemannian manifold and $f: M \rightarrow N$ a harmonic map from $M$ to a complete Riemannian manifold $N$ of nonpositive sectional curvature. Assume that $f$ is of finite energy or that $f$ has bounded image.

If a point $o \in N$ satisfies

$$
\int_{f^{-1}(C(o))} \Delta_{M}(r \circ f)<\infty
$$

then

$$
\int_{f^{-1}(C(o))} \Delta_{M}(r \circ f)=\int_{M \backslash f^{-1}(C(o))} \Delta_{M}(r \circ f) d v
$$

holds. Here $C(o)$ is the cut locus at $o$ and $r(x)=d_{N}(o, x)$ is the Riemannian distance from o to $x$.

From the above result we immediately have the following.

COROLLARY 5. Let $f$ be a harmonic map from a parabolic manifold $M$ to a complete Riemannian manifold $N$ with Sect $_{N} \leq 0$. If $f$ is of finite energy or $f$ has bounded image and there exists a point $o \in N$ such that $\operatorname{Cap}\left(f^{-1}(C(o))\right)=0$, then $f$ is constant.

Our method enables us to extend these results to maps lacking regularity, which are called finely harmonic maps.

We also obtain a 'monotone property' for proper harmonic maps using these method (Corollary 36). We add some Liouville type theorems on parabolic manifolds in Section 4.

We would remark in the last section a simple argument about our Theorem 20 in the Dirichlet form setting. This was noted by Professor M. Takeda. Such a generalization would allow us to obtain results similar to those presented here in a much wider class of spaces and operators including the cases of jump processes.

\section{Preliminaries and setting.}

1.1. $L$-diffusion and related measures. Let $M$ be a manifold with an elliptic operator $L$ associated with an irreducible, strongly local and regular Dirichlet space $(\mathcal{E}, \mathcal{F})$ on $L^{2}(M, m)$, where $m$ is a Radon measure on $M . L$ and $(\mathcal{E}, \mathcal{F})$ are related to each other in such a way that

$$
-\int_{M} L u v d m=\mathcal{E}(u, v) \quad \text { for } u, v \in \operatorname{Dom}(L) .
$$


It is well-known that a diffusion process $\left(X, P_{x}, x \in M\right)$ uniquely corresponds to $(\mathcal{E}, \mathcal{F})$ (cf. [13]). This diffusion has $L$ as its generator, so we sometimes call this diffusion the $L$ diffusion. By the capacity Cap we mean the capacity defined by this Dirichlet form. We remark that if a Borel set $A$ is of capacity zero, then $A$ cannot be hit by $X$, namely, $A$ is a polar set of $X$.

A Radon measure $\mu$ is said to be smooth, roughly speaking, if $\mu$ does not charge sets of capacity zero. Any Radon measure is decomposed into a smooth measure and a nonsmooth measure which is supported by a set of capacity zero (cf. $[3,14]$ ). Such a smooth measure is called the smooth part of the original measure. In this note $\tilde{\mu}$ usually denotes the smooth part of a measure $\mu$. We also recall Revuz's correspondence between positive continuous additive functionals and smooth measures. It is known that for any positive continuous additive functional $A$, there uniquely exists a smooth measure $\mu$ such that

$$
\int_{M} \phi(x) d \mu(x)=\lim _{t \rightarrow 0} \frac{1}{t} \int_{M} E_{x}\left[\int_{0}^{t} \phi\left(X_{s}\right) d A_{s}\right] d m(x) \text { for } \phi \in C_{0}(M) .
$$

Such a $\mu$ is called the Revuz measure of $A$. If $A_{t}=\int_{0}^{t} h\left(X_{S}\right) d s$, then its Revuz measure is $h(x) d m(x)$. If $u \in \mathcal{F}_{\text {loc }}$, then $u(X)$ has the Fukushima decomposition, namely,

$$
\tilde{u}\left(X_{t}\right)-\tilde{u}\left(X_{0}\right)=M_{t}+A_{t},
$$

where $\tilde{u}$ is a quasi-continuous modification of $u, M$ is a local martingale and $A$ is a continuous additive functional of zero energy. The quadratic variation $\langle M\rangle$ is also a positive continuous additive functional of $X$ and its Revuz measure is denoted by $\mu_{\langle u, u\rangle} \cdot \mu_{\langle u, v\rangle}$ can be defined similarly. It is known that the Dirichlet form $\mathcal{E}(u, u)$ can be written as

$$
\mathcal{E}(u, u)=\frac{1}{2} \int_{M} d \mu_{\langle u, u\rangle} .
$$

If $u$ is a smooth function and $X$ is a Brownian motion on a Riemannian manifold $M$, then

$$
u\left(X_{t}\right)-u\left(X_{0}\right)=M_{t}+\frac{1}{2} \int_{0}^{t} \Delta_{M} u\left(X_{s}\right) d s
$$

and $M_{t}=B\left(\int_{0}^{t}|\nabla u|^{2}\left(X_{s}\right) d s\right)$, where $B$ is the one-dimensional standard Brownian motion. In this case $d \mu_{\langle u, u\rangle}=|\nabla u|^{2}(x) d v(x)$.

1.2. $\delta$ - $L$-subharmonic function. We say that $u$ is an $L$-subharmonic function on $M$ if $u \in \dot{\mathcal{F}}_{\text {loc }}$ (see [13] for the definition and the properties) and

$$
-\infty<\mu_{\langle u, \phi\rangle} \leq 0 \text { for any } \phi \in C_{0} \cap \mathcal{F} \text { and } \phi \geq 0 .
$$

We say that $u$ is an $L$-superharmonic function on $M$ if $-u$ is $L$-subharmonic on $M$ and $u$ is an $L$-harmonic function if $u$ is $L$-superharmonic and $L$-subharmonic. When $L=(1 / 2) \Delta_{M}$ is half of the Laplacian on $M$ and $d m=d v$ is the Riemannian volume on $M$, we have

$$
\mathcal{E}(u, u)=\frac{1}{2} \int_{M}|\nabla u|^{2} d v,
$$


and $X$ is a Brownian motion on $M$. In this case the above definition can be restated as follows. $u$ is a $\Delta_{M}$-subharmonic function (subharmonic function, for short) on $M$ if there exists a closed set $N$ with $\operatorname{Cap}(N)=0$ such that $u$ is in $H_{0, \text { loc }}^{1}(M \backslash N)$ and

$$
-\infty<\int_{M}\langle\nabla u, \nabla \phi\rangle d v \leq 0 \text { for any } \phi \geq 0 \text { and } \phi \in C_{o}^{\infty}(M) .
$$

This class includes all subharmonic functions in the classical sense. An important example of classical subharmonic functions is $u(z)=\log |z|$ for $M=\boldsymbol{C}, L=\Delta$. In this case $u \notin$ $H_{0, \text { loc }}^{1}\left(\boldsymbol{R}^{2}\right)$ but $u$ is a $\Delta$-subharmonic function in our sense.

We say that $u$ is a $\delta$ - $L$-subharmonic function on $M$ if $u$ can be expressed locally as a difference of two $L$-subharmonic functions (cf. [17]).

LEMMA 6. If $u$ is an L-subharmonic function, there is a unique nonnegative measure on $M$ that corresponds to $u$. This measure is called the Riesz measure of $u$ (cf. [16]).

Proof. Let $I(\phi)=-\mu_{\langle u, \phi\rangle}$ for $\phi \in C_{0} \cap \mathcal{F}$. Take a domain $D \subset M$ such that supp $\phi \subset D$ and $\psi \in C_{0}(M)$ such that $\psi=1$ on $D$. The derivation property of the Revuz measure (cf. [13]) implies that

$$
-\mu_{\langle u, \phi\rangle}=-\mu_{\langle u, \psi \phi\rangle} \leq-\|\phi\|_{\infty} \mu_{\langle u, \psi\rangle} .
$$

The regularity of the Dirichlet space means that $C_{0} \cap \mathcal{F}$ is dense in $C_{0}(M)$. Hence, $I(\phi)$ can be extended to a positive continuous functional on $C_{0}(M)$. The Riesz-Markov theorem implies that there exists a unique measure $v_{u}$ such that

$$
I(\phi)=\int_{M} \phi d v_{u} \quad \text { for } \phi \in C_{o}(M) .
$$

From now on $v_{u}$ denotes the Riesz measure of $u$.

If $u$ is a $\delta$ - $L$-subharmonic function on $M$, there a signed measure corresponds to $u$. It is called the Riesz charge, denoted by

$$
v_{u}=v_{1}-v_{2},
$$

where $v_{1}, v_{2}$ are the smallest measures such that the decomposition holds. By the choice of these $v_{1}, v_{2}$ this decomposition is unique.

An important probabilistic characterization of $\delta$ - $L$-subharmonic function $u$ is the fact that $u(X)$ is a semi-martingale for an $L$-diffusion $X$ (cf. [10, 13]). Namely, if $u$ is a $\delta$ - $L$ subharmonic function, then $u(X)$ can be decomposed as

$$
\tilde{u}\left(X_{t}\right)-\tilde{u}\left(X_{0}\right)=M_{t}+A_{t},
$$

where $\tilde{u}$ is a quasi-continuous modification of $u, M$ is a local martingale and $A$ is a continuous additive functional of bounded variation. We remark that any $u \in \dot{\mathcal{F}}_{\text {loc }}$ has a quasi-continuous modification $\tilde{u}$. The Revuz measure corresponding to $A$ is

$$
\tilde{v}_{u}=\tilde{v}_{1}-\tilde{v}_{2}
$$


where $\tilde{v}_{1}$ and $\tilde{v}_{2}$ are the smooth parts of $v_{1}$ and $v_{2}$ and $v_{u}=v_{1}-v_{2}$ is the Riesz measure of $u$. For a general $\delta$-L-subharmonic function $u$ we define $\mu_{\langle u, u\rangle}$ as follows. Let $\left\{G_{n}\right\}$ be a sequence of finely open sets of $M$ such that $G_{n} \subset G_{n+1}, u=u_{n}, m$-almost everywhere on $G_{n}$ for some $u_{n} \in \mathcal{F}$ and $\operatorname{Cap}\left(M \backslash \bigcup_{n} G_{n}\right)=0$. Note that $\left\langle M^{T_{n}}\right\rangle_{t}$ is a positive continuous additive functional where $M^{T_{n}}$ is stopped by $T_{n}=\inf \left\{t>0 ; u\left(X_{t}\right) \notin G_{n}\right\}$, since $u$ on $G_{n}$ belongs to $\mathcal{F}_{\text {loc }}$.

Let $\mu_{\langle u, u\rangle}^{(n)}$ be the Revuz measure of $\left\langle M^{T_{n}}\right\rangle_{t}$. Set $\mu_{\langle u, u\rangle}=\sup _{n} \mu_{\langle u, u\rangle}^{(n)}$. We say that $u$ is of finite Dirichlet integral or finite ( $L$-)energy if $\mu_{\langle u, u\rangle}(M)=\sup _{n} \mu_{\langle u, u\rangle}^{(n)}(M)<\infty$.

If $\mu_{\langle u, u\rangle}$ is absolutely continuous with respect to $m$, then its density $d \mu_{\langle u, u\rangle} / d m$ is denoted by $\Gamma(u, u)$ and is called the 'carré du champ' of $u$.

We proceed to the stochastic calculus for $\delta$ - $L$-subharmonic functions.

LEMMA 7 (Tanaka's formula cf. [24]). Let $Y$ be a semi-martingale on $\boldsymbol{R}$ and $a \in \boldsymbol{R}$.

(ii)

$$
Y_{t} \wedge a=Y_{0} \wedge a+\int_{0}^{t} 1_{\left\{Y_{s} \leq a\right\}} d Y_{s}-\frac{1}{2} L_{t}^{a},
$$

$$
Y_{t} \vee a=Y_{0} \vee a+\int_{0}^{t} 1_{\left\{Y_{s} \geq a\right\}} d Y_{s}+\frac{1}{2} L_{t}^{a} .
$$

An increasing process $L^{a}$ is called a local time at a of $Y$.

If $Y=u(X)$, then $L^{a}$ is a positive continuous additive functional. We define the smooth measure $l_{u}^{a}$ on $M$ as the Revuz measure of $L^{a}$ with $Y=u(X)$.

From this we have the following.

PROPOSITION 8. If $u$ is $a \delta$-L-subharmonic function, then $u \wedge a$ and $u \vee a$ are $\delta$ - $L$ subharmonic functions for $a \in \boldsymbol{R}$. Their Riesz charges are

$$
d v_{u \wedge a}=1_{\{u \leq a\}} d v_{u}-(1 / 2) l_{u}^{a} \quad \text { and } \quad d v_{u \vee a}=1_{\{u \geq a\}} d v_{u}+(1 / 2) l_{u}^{a} .
$$

In particular, if $u=u^{+}-u^{-}$, where $u^{-}, u^{+}$are the positive and negative parts of $u$, respectively, then

$$
d v_{u^{-}}=-1_{\{u \leq 0\}} d v_{u}+(1 / 2) l_{u}^{a} \quad \text { and } \quad d v_{u^{+}}=1_{\{u \geq 0\}} d v_{u}+(1 / 2) l_{u}^{a} .
$$

We give the $\delta$-subharmonic version of the coarea formula.

PROpOSITION 9. Let $u$ be a $\delta$-L-subharmonic function satisfying $\mu_{\langle u, u\rangle}(\{u \leq r\})<$ $\infty$ for each $r \in \boldsymbol{R}$. Then for $\phi \in C_{o}(\boldsymbol{R})$ and $\psi \in C_{o}(M)$ we have

$$
\int_{M} \phi(u(x)) \psi(x) d \mu_{\langle u, u\rangle}(x)=\int_{\boldsymbol{R}} \int_{M} \phi(y) \psi(x) d l_{u}^{y}(x) d y .
$$

Proof. We may assume that $\phi \geq 0, \psi \geq 0$. Recall that the occupation time formula for a semi-martingale $Y$ (cf. [24]):

$$
\int_{0}^{t} \phi\left(Y_{s}\right) d\langle Y, Y\rangle_{s}=\int_{\boldsymbol{R}} \phi(y) L_{t}^{y} d y \quad \text { for } \phi \in C_{o}(\boldsymbol{R}) .
$$


Setting $Y=u(X)$ and integrating both sides with respect to $d P_{x} \cdot \psi(x) d m(x)$, we have

$$
\int_{M} \psi(x) E_{x}\left[\int_{0}^{t} \phi\left(u\left(X_{s}\right)\right) d\langle u(X), u(X)\rangle_{s}\right] d m(x)=\int_{M} \psi(x) E_{x}\left[\int_{\boldsymbol{R}} \phi(y) L_{t}^{y} d y\right] d m(x) .
$$

Since the limit in the definition of the Revuz measure is monotone, we have the desired equality.

From Proposition 9 we have the following.

COROLlary 10. Let $M$ be a complete Riemannian manifold.

(i) Let $u$ be a proper $C^{2}$-function and $X$ a Brownian motion on $M$. The local time measure $l_{u}^{r}(M)$ on $u^{-1}(r)$ is given by

$$
l_{u}^{r}(M)=\int_{u^{-1}(r)}\|\nabla u\|(x) d A(x) \quad \text { for almost every } r,
$$

where $d A$ is the induced volume form on $u^{-1}(r)$.

(ii) Suppose $\operatorname{dim} M \geq 2$. Let $u(x)=d_{M}(o, x)$ be the Riemannian distance function for $o \in M$. Then $l_{u}^{r}(M)$ equals the ( $\left.\operatorname{dim} M-1\right)$-dimensional Hausdorff measure of $\partial B(r)$, where $\partial B(r)=\left\{x \in M ; d_{M}(o, x)=r\right\}$.

Proof. (i) Note that $d \mu\langle u, u\rangle=\|\nabla u\|^{2} d v$, where $d v$ is the Riemannian volume measure.

(ii) It is well-known that the cut locus of $o C(o)$ is a null set with respect to $d v$. Then $\|\nabla u\|=1 d v$-almost everywhere. Hence $l_{u}^{r}(M)=d V(r) / d r$ almost every $r$, where $V(r)$ is the Riemannian volume of $B(r)=\left\{x \in M ; d_{M}(o, x)<r\right\}$. On the other hand, the coarea formula for Lipschitzian functions (cf. [9]) implies that $d V(r) / d r$ equals the $(\operatorname{dim} M-1)$ dimensional Hausdorff measure of $\partial B(r)$.

We put the following Assumptions 1 and 2 on $L$ for simplicity in some cases. We need these assumptions only for Theorem 12.

ASSUMPTION 1. If $D$ is a relatively compact domain of $M$, then there exists a Green function $g_{D}(x, y)$ on $D$ with Dirichlet boundary condition satisfying

$g_{D}(x, y)>0$ for $x, y \in D$ and $g_{D}(x, y)$ is continuous for $x, y \in D \times D \backslash$ the diagonal.

ASSUMPTION 2. Any $L$-subharmonic function satisfies the Riesz decomposition formula, namely, if $u$ is an $L$-subharmonic function on $M$ and $D$ is a relatively compact domain in $M$, then there exists a $L$-harmonic function $h$ on $D$ bounded on $\bar{D}$ such that

$$
u(x)=h(x)-\int_{D} g_{D}(x, y) d v_{u}(y)
$$

holds outside a set of zero capacity.

REMARK 11. Assumptions 1 and 2 are satisfied by Brownian motions on Riemannian manifolds and diffusion processes with uniformly elliptic generators. 
We note the following about the smooth parts of Riesz measure of $\delta$ - $L$-subharmonic functions.

THEOREM 12. Let u be a $\delta$-L-subharmonic function on $M$ satisfying that $\mu_{\langle u, u\rangle}(M)<$ $\infty$ or $u$ is bounded. Then

$$
\tilde{v}_{u}=v_{u}
$$

Namely, the Riesz charge of u has no nonsmooth part.

REMARK 13. Under the assumption $\mu_{\langle u, u\rangle}(M)<\infty$, the conclusion holds without Assumptions 1 and 2 in the framework of Dirichlet spaces. We come back to this point in the Appendix.

Proof of TheOREM 12. Let $D$ be a relatively compact domain of $M$. It suffices to show the result for $D$ instead of $M$. Let $u=u_{1}-u_{2}$, where $u_{1}, u_{2}$ are $L$-subharmonic functions. It suffices to show the assertion for each $u_{k}, k=1,2$. Thus, we assume that $u$ is an $L$-subharmonic function. Set $u=u^{+}-u^{-}, u^{+}, u^{-} \geq 0$. Then $u^{+}, u^{-}$are $\delta$ - $L$-subharmonic functions. Since $l_{u}$ is smooth, by Proposition 8 ,

$$
d \tilde{\nu}_{u^{-}}-d v_{u^{-}}=1_{\{u \leq 0\}} d\left(v_{u}-\tilde{v}_{u}\right) .
$$

On the other hand, $u(X)$ is a semi-martingale. Then

$$
u^{-}\left(X_{t}\right)-u^{-}\left(X_{0}\right)=M_{t}+A_{t}
$$

where $M$ is a local martingale and $A$ is a process of bounded variation. Integrating both sides with the stopping time argument, we have

$$
E_{x}\left[u^{-}\left(X_{\tau_{D}}\right)\right]-u^{-}(x)+N_{x}\left(\tau_{D}, u^{-}\right)=E_{x}\left[A_{\tau_{D}}\right]=\int_{D} g_{D}(x, y) d \tilde{v}_{u^{-}},
$$

where

$$
N_{x}\left(\tau_{D}, u\right)=\lim _{\lambda \rightarrow \infty} \lambda P_{x}\left(\sup _{0<t<\tau_{D}} u^{-}\left(X_{t}\right)>\lambda\right)
$$

By Assumption 2, the Riesz decomposition of $u^{-}$implies that there exists an $L$-harmonic function $h$ on $D$ such that

$$
u^{-}(x)=h(x)-\int_{D} g_{D}(x, y) d v_{u^{-}}
$$

Hence

$$
N_{x}\left(\tau_{D}, u^{-}\right)=\int_{D} g_{D}(x, y) 1_{\{u \leq 0\}}(y) d\left(v_{u^{-}}-\tilde{v}_{u^{-}}\right)(y) .
$$


We claim that if $\mu_{\langle u, u\rangle}(M)<\infty$ or $u$ is bounded, then $N_{x}\left(\tau_{D}, u^{-}\right)=0$. If $u$ is bounded, then the result is obvious. Assume that $\mu_{\langle u, u\rangle}(M)<\infty$. If $\lambda$ is large enough, then

$$
\begin{aligned}
P\left(\sup _{0<t<\tau_{D}} u^{-}\left(X_{t}\right)>\lambda\right) & \leq P\left(\sup _{0<t<\tau_{D}}\left|M_{t}\right|+A_{\tau_{D}}+u^{-}(x)>\lambda\right) \\
& \leq P\left(\sup _{0<t<\tau_{D}}\left|M_{t}\right|>\frac{\lambda}{2}\right)+P\left(A_{\tau_{D}}>\frac{\lambda}{2}\right) .
\end{aligned}
$$

By the Burkholder inequality, for a constant $c>0$

$$
P_{x}\left(\sup _{0<t<\tau_{D}}\left|M_{t}\right|>\frac{\lambda}{2}\right) \leq \frac{c}{\lambda^{2}} E_{x}\left[\langle M\rangle_{\tau_{D}}\right] .
$$

The assumption implies that $E_{x}\left[\langle M\rangle_{\tau_{D}}\right]<\infty$ for almost every $x$. With $E_{x}\left[A_{\tau_{D}}\right]<\infty$ we have the desired result. It is easy to see from Proposition 8 that

$$
N_{x}\left(\tau_{D}, u^{+}\right)=0 .
$$

We apply Theorem 12 to complex functions.

COROLLARY 14. Let $f$ be a meromorphic function on a Kähler manifold $M$ and $D$ a relatively compact domain in $M$. If there exists a constant $c>0$ such that

$$
\int_{D \cap\{|f|>c\}} \frac{|d f|^{2}}{|f|^{2}} d v<\infty,
$$

then $f$ is holomorphic on $D$ and never vanishes on $D$.

We note a related result due to Fukushima in the theory of Dirichlet spaces.

Proposition 15 (Fukushima [11]). The Riesz measure of a $\delta$-L-subharmonic function in $\mathcal{F}_{\text {loc }}$ is smooth.

1.3. $L$-finely harmonic maps and their energy. If $N$ is a manifold with a connection, we can naturally define a geodesic. We say that a function $h$ on $N$ is geodesically convex if $t \mapsto h(\gamma(t))$ is convex for any geodesic $\gamma$.

Definition 16 (cf. [8]). A continuous stochastic process $Y$ on a probability space $\left(\Omega, \mathcal{F}, \mathcal{F}_{t}, P\right)$ is called a martingale on a manifold $N$ if for any geodesically convex function $v$ defined on each open subset $U \subset N, v(Y)$ is a submartingale with respect to $\left(\Omega, \mathcal{F}, \mathcal{F}_{t}, P\right)$ when $Y$ lies in $U$.

Definition 17. A Borel measurable map $f: M \rightarrow N$ is called an $L$-finely harmonic map if $f(X)$ is a martingale on $N$ for the $L$-diffusion $X$.

From now on we assume that the target manifold $N$ is a Riemannian manifold. Then we can define the intrinsic time of a martingale $Y$ on $N$. 
DEFINITION 18. Let $\tilde{Y}$ be the stochastic development of $Y$ (cf. [8, 12]). We define the intrinsic time $[Y, Y]$ by

$$
d[Y, Y]_{t}=\sum_{i} d\left\langle\tilde{Y}^{i}, \tilde{Y}^{i}\right\rangle_{t}
$$

where $\langle\cdot, \cdot\rangle$ is the usual quadratic variation for one-dimensional local martingales.

We remark that if $Y=f(X)$, then $[Y, Y]$ is a positive continuous additive functional of $X$. These facts enable us to define the energy of $L$-finely harmonic map.

Definition 19. Let $f: M \rightarrow N$ be an $L$-finely harmonic map and $X$ an $L$-diffusion on $M$. We define an energy measure $E_{f}$ of $f$ as the Revuz measure of the intrinsic time $[f(X), f(X)]$ of $f(X)$ (if necessarily, via the stopping time argument). Namely,

$$
\int_{M} \phi(x) d E_{f}(x)=\lim _{t \rightarrow 0} \frac{1}{t} \int_{M} E_{x}\left[\int_{0}^{t} \phi\left(X_{s}\right) d[f(X), f(X)]_{s}\right] d m(x) \text { for } \phi \in C_{0}(M) \text {. }
$$

If $d E_{f}(x)$ is absolutely continuous with respect to $d m$, then $e_{f}$ denotes the density.

2. A divergence theorem for some $\delta$ - $L$-subharmonic functions on parabolic manifolds. We say that a manifold $M$ is $L$-recurrent (resp. $L$-transient) if $M$ admits a recurrent (resp. transient) $L$-diffusion. Our basic observation is as follows.

THEOREM 20. The following conditions are equivalent.

(i) $M$ is L-recurrent.

(ii) For any $\delta$-L-subharmonic function $u$ on $M$ with $\mathcal{E}(u, u)<\infty$, the positive and negative smooth parts $\tilde{v}^{+}$and $\tilde{v}^{-}$of the Riesz charge of u satisfy

$$
\tilde{v}^{-}(M)=\tilde{v}^{+}(M) \quad(\leq \infty) .
$$

(iii) For any bounded $\delta$-L-subharmonic function $u$ on $M$, the positive and negative smooth parts $\tilde{v}^{+}$and $\tilde{v}^{-}$of the Riesz charge of u satisfy

$$
\tilde{v}^{-}(M)=\tilde{v}^{+}(M) \quad(\leq \infty) .
$$

REMARK 21. In cases (ii) and (iii) of the above theorem

$$
v^{-}(M)=\tilde{v}^{-}(M)=\tilde{v}^{+}(M)=v^{+}(M)
$$

holds by Theorem 12 .

The above assertion (i) $\Rightarrow$ (ii) can be strengthened as follows.

THEOREM 22. Let $M$ be an L-recurrent manifold and $u$ a $\delta$-L-subharmonic function on $M$ with $\mu_{\langle u, u\rangle}(M)<\infty$. We also assume that $u$ is finely continuous and upper semicontinuous.

Then either the positive and negative smooth parts $\tilde{v}^{+}$and $\tilde{v}^{-}$of the Riesz charge of $u$ satisfy

$$
0<\tilde{v}^{-}(M)=\tilde{v}^{+}(M) \quad(\leq \infty),
$$


or $u$ is constant.

REMARK 23. If the continuity assumption of the above theorem is removed, the conclusion becomes that either $0<\tilde{v}^{-}(M)=\tilde{v}^{+}(M)(\leq \infty)$ or $u$ is constant $m$-almost everywhere.

We use a ratio ergodic theorem for recurrent Markov processes for the proof of Theorem 22.

Lemma 24 (cf. [24]). Let $C^{(1)}, C^{(2)}$ be positive continuous additive functionals of $X$ and $\mu_{1}, \mu_{2}$ the corresponding Revuz measure, respectively. Assume that $X$ is recurrent and $\mu_{2}(M)<\infty$. Then we have

$$
\lim _{t \rightarrow \infty} \frac{C_{t}^{(1)}}{C_{t}^{(2)}}=\frac{\mu_{1}(M)}{\mu_{2}(M)} \quad \text { almost surely. }
$$

Proof of Theorem 22. By the Fukushima decomposition or Itô's formula we have

$$
u\left(X_{t}\right)-u\left(X_{0}\right)=M_{t}+A_{t}^{(1)}-A_{t}^{(2)},
$$

where $M$ is a local martingale and $A^{(1)}, A^{(2)}$ are positive continuous additive functionals corresponding to the smooth measures $\tilde{v}^{+}, \tilde{v}^{-}$, respectively. Recall that $M_{t}=B\left(\langle M\rangle_{t}\right)$ with a one-dimensional Brownian motion $B$ and the Revuz measure of $\langle M\rangle$ is $\mu\langle u, u\rangle$. The assumption implies that $\mu_{\langle u, u\rangle}(M)<\infty$. We note that by the assumption and the above lemma,

$$
\lim _{t \rightarrow \infty} \frac{A_{t}^{(1)}}{\langle M\rangle_{t}}=\frac{\tilde{v}^{+}(M)}{\mu_{\langle u, u\rangle}(M)} \quad(\leq \infty), \quad \lim _{t \rightarrow \infty} \frac{A_{t}^{(2)}}{A_{t}^{(1)}}=\frac{\tilde{v}^{-}(M)}{\tilde{v}^{+}(M)}
$$

Combining this with the law of iterated logarithm of the one-dimensional Brownian motion

$$
\limsup _{t \rightarrow \infty} \frac{\left|B_{t}\right|}{\sqrt{2 t \log \log t}}=1, \quad \text { almost surely }
$$

we have

$$
\begin{array}{llll}
\text { if } \tilde{v}^{-}(M)<\tilde{v}^{+}(M), & u\left(X_{t}\right) \rightarrow+\infty & \text { as } t \rightarrow \infty & \text { almost surely } \\
\text { if } \tilde{v}^{-}(M)>\tilde{v}^{+}(M), & u\left(X_{t}\right) \rightarrow-\infty & \text { as } t \rightarrow \infty & \text { almost surely. }
\end{array}
$$

This contradicts the assumption that $X$ is recurrent. Hence, $\tilde{v}^{-}(M)=\tilde{v}^{+}(M)$. If $\tilde{v}^{-}(M)=0$, then $u$ is harmonic, and $u$ takes the form of

$$
u\left(X_{t}\right)-u\left(X_{0}\right)=M_{t} \quad \text { with } \quad M_{t}=B\left(\langle M\rangle_{t}\right) .
$$

We consider $U_{t}=\max \left\{M_{t}, 0\right\}$. Tanaka's formula (cf. [24]) implies that

$$
U_{t}=\int_{0}^{t} 1_{M_{s}>0} d M_{s}+l_{t},
$$

where $l_{t}$ is the local time at 0 of $M_{t} . l_{t}$ is also a positive continuous additive functional of $X$. The Revuz measure corresponding to the quadratic variation $\left\langle\int_{0}^{t} 1_{M_{S}>0} d M_{S}\right\rangle=$ $\int_{0}^{t} 1_{M_{s}>0} d\langle M\rangle_{s}$ is finite since that corresponding to $\langle M\rangle$ is finite by assumption. We again use 
the above ratio ergodic theorem, and get $l_{t}=0$. It implies that $u$ is lower or upper bounded. The recurrence implies that $u$ should be constant.

Proof of (ii) $\Rightarrow$ (i) IN TheOREM 20. Assume that $M$ is not $L$-recurrent. In general (cf. [13]) this assumption implies that there exists $h \in L_{+}^{1}(m)$ such that $G h \in \mathcal{F}_{e}$ with $\mu_{\langle G h, G h\rangle}(M)<\infty$ and $-G h$ is $L$-subharmonic where $G$ is the zeroth resolvent operator, a contradiction. In the case of a Brownian motion the situation is more familiar. The assumption implies that there exists a Green function $g$ on $M$. For a positive $\phi \in C_{0}^{\infty}(M)$, let

$$
v(x)=\int_{M} g(x, y) \phi(y) d v_{M}(y) .
$$

Then $v$ is a superharmonic function with finite Dirichlet integral.

ProOF OF (iii) $\Rightarrow$ (i) IN THEOREM 20. If $M$ is not $L$-recurrent, then there exists a nonconstant, bounded $L$-subharmonic function which is not $L$-harmonic. This is impossible by (iii).

ProOF OF (i) $\Rightarrow$ (iii) IN THEOREM 20. We can show more generally the following theorem.

THEOREM 25. Let $M$ be an L-recurrent manifold. Suppose that a $\delta$-L-subharmonic function $u$ is bounded from below. If there exists $r>\inf u$ such that $\tilde{v}^{+}(\{u \leq r\})<\infty$, then

$$
\tilde{v}^{+}(\{u \leq r\})=\tilde{v}^{-}(\{u \leq r\})+l_{u}^{r},
$$

where $l_{u}^{r}$ is the smooth measure corresponding to the local time at $\{u=r\}$ of $X$.

REMARK 26. The assumption that the lower boundedness of $u$ is not essential and only for simplicity.

This immediately follows from Tanaka's formula and the ratio ergodic theorem of the following type.

LEMMA 27 (cf. [24]). Let $C^{(1)}, C^{(2)}$ be positive continuous additive functionals of $X$ and $\mu_{1}, \mu_{2}$ the corresponding Revuz measure, respectively. Assume that $X$ is recurrent and $\mu_{2}(M)<\infty$. Then we have

$$
\lim _{t \rightarrow \infty} \frac{E_{x}\left[C_{t}^{(1)}\right]}{E_{x}\left[C_{t}^{(2)}\right]}=\frac{\mu_{1}(M)}{\mu_{2}(M)}, \quad P_{x} \text {-almost surely, } \quad m \text {-almost everywhere } x \in M .
$$

In connection with Takegoshi's result we give a similar and slightly more general result here. Let $C(K, G)$ be the condenser capacity defined by

$$
C(K, G)=\mathcal{E}\left(e_{K, G}, e_{K, G}\right),
$$

where $e_{K, G}$ is the equilibrium potential which is a unique element of $\mathcal{F}$ satisfying $e_{K, G}=1$ on $K, e_{K, G}=0$ on $G^{c}$ and $e_{K, G}$ harmonic on $G \backslash \bar{K}$.

PROPOSITION 28. If there exists an exhaustion $\left\{G_{n}\right\}$ satisfying

$$
\mu_{\langle u, u\rangle}\left(G_{n}\right) C\left(G_{1}, G_{n}\right) \rightarrow 0 \text { as } n \rightarrow \infty,
$$


then

$$
\tilde{v}^{-}(M)=\tilde{v}^{+}(M) \text {. }
$$

It is easy to check that in the Riemannian case

$$
C(B(1), B(r)) \leq \text { constant }\left(\int_{1}^{r} \frac{d t}{V^{\prime}(t)}\right)^{-1} \text {. }
$$

\section{Applications.}

3.1. Harmonic maps from $L$-recurrent manifolds. We apply these divergence theorems to harmonic maps. Let $f: M \rightarrow N$ be an $L$-finely harmonic map and $N$ a Riemannian manifold. Fix an arbitrary reference point $o \in N$. Set $r(x)=d(o, x)$, where $d$ is the Riemannian distance on $N$. Then $r(x)$ is a smooth function on $N \backslash(C(o) \cup\{o\})$, where $C(o)$ is the cut locus of $o$. It is well known that $-\Delta r$ is a nonnegative distribution on $C(o)$ (cf. Appendix in [26]). Cranston et al. [6] gave a probabilistic counterpart to this in martingale language. Namely, they showed that if $Y$ is a martingale on $N$ and $Y$ does not hit $o$, then

$$
r\left(Y_{t}\right)-r\left(Y_{0}\right)=\text { a local martingale }+\frac{1}{2} \int_{0}^{t} 1_{N \backslash C(o)} \operatorname{Hess} r(d Y, d Y)-L_{t},
$$

where Hess is the Hessian with respect to the Levi-Civita connection of $N$ and $L$ is an increasing process which increases only when $Y$ stays on $C(o)$. From this we can see that $r \circ f$ is a $\delta$ - $L$-subharmonic function on $M$ and $r$ is a $\delta$ - $\Delta_{N}$-subharmonic function on $N$.

It is well known that $-\left.\Delta r\right|_{C(o)}$ is singular to the Riemannian volume measure. We apply Theorem 12 or Proposition 15 to the cases where $Y$ is a Brownian motion on $N$ and $Y=f(X)$ for an $L$-finely harmonic map $f: M \rightarrow N$ with an $L$-diffusion $X$, and obtain the following.

PROPOSITION 29. In the notation as above, we have:

(i) $-\left.\Delta r\right|_{C(o)}$ is a smooth measure;

(ii) if $f: M \rightarrow N$ is locally bounded or the energy of $f$ is locally finite, then $-\Delta r \circ$ $\left.f\right|_{f^{-1}(C(o))}$ is a smooth measure.

If $\operatorname{Sect}_{N} \leq 0$, then $r(x)$ is a convex function outside $C(o)$. Hence, when $Y=f(X)$, the last two terms in the above equation due to Cranston et al. are positive continuous additive functionals of $X$. Their Revuz measure are the smooth parts of the positive part $\mu_{1}$ and the negative part $\mu_{2}$ of the Hahn decomposition of $L(r \circ f)$, respectively.

The following is a direct consequence of Theorem 22.

COROLlary 30. Assume that $M$ is an L-recurrent manifold, $N$ is a Riemannian manifold and $f: M \rightarrow N$ is an L-finely harmonic map. Let $\mu_{1}, \mu_{2}$ be as above. If $f$ is of finite $L$-energy and there exists a point $o \in N$ such that $\operatorname{Cap}\left(f^{-1}(C(o))\right)=0$ and

$$
\tilde{\mu}_{2}(M)<\infty,
$$

then

$$
0<\tilde{\mu}_{2}(M)=\tilde{\mu}_{1}(M) \text { or } f \text { is constant q.e. }
$$


In particular if $\tilde{\mu}_{2}(M)=0$, then $f$ is constant q.e.

Proof. We have only to note that if $f$ is of finite $L$-energy, then $\mu_{\langle r \circ f, r \circ f\rangle}(M)<\infty$. $|\nabla r| \leq 1$ and $d r \otimes d r \leq g_{N}$ imply that

$$
\langle M\rangle_{t} \leq[f(X), f(X)]_{t},
$$

where $M$ is the martingale part of $r \circ f(X)$. It follows from this that

$$
d \mu\langle r \circ f, r \circ f\rangle \leq d E_{f} .
$$

3.2. Green's formula. Theorem 25 can be regarded as a generalization of Green's formula for $\delta$ - $L$-subharmonic functions. We can recover the usual Green's formula for smooth functions from Theorem 25 and stochastic calculus.

PROPOSITION 31. (i) If $\phi$ is a smooth function on a complete Riemannian manifold $M$ and $D$ is a relatively compact domain with smooth boundary $\partial D$ in $M$, then

$$
\frac{1}{2} \int_{D} \Delta_{M} \phi(x) d v(x)=\int_{\partial D} \frac{\partial \phi}{\partial n} d A,
$$

where $\partial / \partial n$ is the outward normal derivative on $\partial D$.

(ii) Let $u$ be a proper $C^{2}$-function on a complete Riemannian manifold $M$. Then for almost every $r \in(0, \infty)$, we have

$$
\int_{\{u \leq r\}} \Delta_{M} u(x) d v(x)=\int_{u^{-1}(r)}\|\nabla u\|(x) d A(x) .
$$

Proof. (i) We consider a Brownian motion $\tilde{X}$ on $\bar{D}$ reflecting at $\partial D$. It is known that such a process can be constructed on $\bar{D}$ since $\partial D$ is smooth. Since $\tilde{X}$ is recurrent, the previous argument is applicable. Namely, we regard $\bar{D}$ as a parabolic manifold. $\tilde{X}$ has a generator $L=(1 / 2) \Delta_{M}$ with Neumann boundary condition. Moreover, it has a form in SDE using the original Brownian motion $X$ on $M$ as

$$
d \tilde{X}=d X-n(\tilde{X}) d L,
$$

where $L$ is the local time of $X$ on $\partial D$ and $n$ is the outward normal vector to $\partial D$. Hence, for a smooth function $\phi$,

$$
\phi\left(\tilde{X}_{t}\right)-\phi\left(\tilde{X}_{0}\right)-\int_{0}^{t} \frac{1}{2} \Delta_{M} \phi\left(\tilde{X}_{s}\right) d s+\int_{0}^{t} \frac{\partial \phi}{\partial n} d L_{t}=\text { a local martingale } .
$$

We also note that the Revuz measure of $L$ is the surface measure on $\partial D$. Since $\phi$ is bounded on $\bar{D}$, Theorem 20(ii) implies the desired formula.

(ii) Let $D$ be a relatively compact domain with smooth boundary $\partial D$ in $M$ such that $\{u \leq r\} \subset D$. A standard argument enables us to apply the above formula formally to $\phi=u \wedge r$. Our choice of $D$ means that $(\partial / \partial n)(u \wedge r)=0$. Tanaka's formula and the same argument as before lead us to the desired formula. 
REMARK 32. We can give a general version of Green's formula such as (i) of the above proposition provided that a reflecting $L$-diffusion exists. We can construct it with some assumptions on $D$ and the diffusion. Since preparations are needed, we omit the detail here.

We note the case where $u(x)=r(x)$ with $r(x)=d_{N}(o, x)$ and $N$ is a complete Riemannian manifold. Let $l$ denote $-\left.\Delta_{N} r\right|_{C(o)}$. By Theorem 25 and Corollary 10 with the argument in the proof of Proposition 31 we have the following.

COROLlary 33. Let $N$ be a complete Riemannian manifold, $o \in N$ an arbitrary point and $C(o)$ the cut locus of $o$. For almost every $r \in(0, \infty)$ with $o \notin B_{r}(a)$ and $a \in N$, we have

$$
\int_{C(o) \cap B_{a}(r)} l+\operatorname{vol}\left(\partial B_{a}(r)\right)=\int_{B_{a}(r) \backslash C(o)} \Delta_{N} r(x) d v(x),
$$

where $B_{a}(r)$ is the geodesic ball with center $a \in N$ and radius $r$, and $\operatorname{vol}\left(\partial B_{a}(r)\right)$ denotes the $(\operatorname{dim} N-1)$-dimensional Hausdorff measure of $\partial B_{a}(r)$ with respect to the Riemannian metric.

3.3. Proper $L$-finely harmonic maps. We apply the results in the previous sections to get a general estimate of the energy of proper maps to Cartan-Hadamard manifolds.

LEMMA 34. Let $u$ be a proper $\delta$-L-subharmonic function such that $d \mu\langle u, u\rangle$ is locally finite and

$$
v_{u} \geq k(u(x)) d \mu\langle u, u\rangle-a(x) d m
$$

for $k \geq 0$ on $[0, \infty)$, where $k(u(x))$ is locally integrable for $d \mu\langle u, u\rangle$ and $a(x)$ is a locally integrable function on $M$ with respect to $\mathrm{dm}$. Then we have

$$
\int_{-\infty}^{r} k(t) l_{u}^{t}\left(u^{-1}(t)\right) d t-\int_{\{u \leq r\}} a(x) d m(x) \leq l_{u}^{r}\left(u^{-1}(r)\right) .
$$

COROLLARY 35. Under the assumption as in the above lemma, we have for $\delta<r$

$$
\begin{aligned}
\int_{\{u \leq r\}} k(u(x)) d \mu\langle u, u\rangle \\
\quad \geq e^{\int_{\delta}^{r} k(t) d t}\left\{\int_{\{u \leq \delta\}} k(u(x)) d \mu\langle u, u\rangle-\int_{\delta}^{r} e^{-\int_{\delta}^{t} k(s) d s} k(t) \int_{u \leq t} a(x) d m(x) d t\right\} .
\end{aligned}
$$

We apply this to proper $L$-finely harmonic maps. Let $N$ be a Cartan-Hadamard manifold and $r(x)=d(o, x)$ the distance function on $N$. If $\phi$ is a $C^{2}$-function on $[0, \infty)$, by direct calculation we have

$$
\operatorname{Hess} \phi(r)=\phi^{\prime}(r) \operatorname{Hess} r+\phi^{\prime \prime}(r) d r \otimes d r .
$$

We also remark that the Hessian comparison theorem (cf. [15]) implies

$$
\text { Hess } r \geq \rho(r)\left(g_{N}-d r \otimes d r\right),
$$

where $g_{N}$ is the Riemannian metric of $N$ and $\rho(t)=1 / t$ if the sectional curvature of $N \leq 0$, and $\rho(t)=\sqrt{\kappa}$ if the sectional curvature of $N \leq-\kappa<0$. 
Combining these observations with the derivation property of Revuz measure, we have the following:

COROLlARY 36. Let $f: M \rightarrow N$ be a proper $L$-finely harmonic map from a manifold $M$ which supports a recurrent $L$-diffusion to a Cartan-Hadamard manifold $N$. Then there exists a constant $C>0$ depending only on $\varepsilon>0$ such that

$$
\int_{f^{-1}\left(B_{o}(r)\right)} \frac{1}{d_{N}(o, f(x))^{1-\varepsilon}} d E_{f}(x) \geq C\left(\frac{r}{\delta}\right)^{\varepsilon} \int_{f^{-1}\left(B_{o}(\delta)\right)} \frac{1}{d_{N}(o, f(x))^{1-\varepsilon}} d E_{f}(x),
$$

for $0<\delta<r<\infty$ and $\varepsilon>0$, where $B_{o}(r)$ is the geodesic ball with center $o$ and radius $r$ in $N$.

Proof. We can set $u=\phi(r \circ f)$. We take $\phi(t)=t^{1+\varepsilon}$. Then $k(t)=\varepsilon /((1+\varepsilon) t)$ in Corollary 35 will do.

3.4. Meromorphic functions. We can also apply our results to meromorphic functions on parabolic Kähler manifolds.

THEOREM 37. Let $f$ be a meromorphic function on a parabolic Kähler manifold $M$. If there exists a constant $c>0$ such that

$$
\int_{\{|f|>c\}} \frac{|d f|^{2}}{|f|^{2}} d v_{M}<\infty
$$

then $f$ is constant.

Proof. Note that $u=\log |f|$ is a $\delta$-subharmonic function on $M$ and the smooth part of its Riesz measure vanishes. We can apply the same argument as in Theorem 22 to $u \vee \log c$ to get the desired result.

It is known that there exist recurrent holomorphic diffusions (see $[12,20]$ ) on $\boldsymbol{C}^{n}$ and submanifolds in $\boldsymbol{C}^{n}$ satisfying a certain volume growth condition (for instance, algebraic submanifolds). $u$ appearing in the above proof is a $\delta$ - $L$-subharmonic function if $L$ is the generator of a holomorphic diffusion. Kaneko [20] gave such a recurrent holomorphic diffusion by the Dirichlet form on $L^{2}\left(\boldsymbol{C}^{n}, d m\right)$ defined by

$$
\mathcal{E}(\phi, \psi)=\int_{\boldsymbol{C}^{n}} d \phi \wedge d^{c} \psi \wedge \omega^{n-1} \text { for } \phi, \psi \in C_{0}^{\infty}\left(\boldsymbol{C}^{n}\right),
$$

and $d m=d d^{c}|z|^{2} \wedge \omega^{n-1}$, where $\omega=d d^{c} \log \left(1+|z|^{2}\right)$. Then our general setting enables us to obtain the following.

THEOREM 38. Let $f$ be a meromorphic function on $\boldsymbol{C}^{n}$ and $d m$ as above. If there exists a constant $c>0$ such that

$$
\int_{\{|f|>c\}} d \log |f| \wedge d^{c} \log |f| \wedge \omega^{n-1}<\infty,
$$

then $f$ is constant. 
4. Other Liouville theorems on $L$-recurrent manifolds. This section is additional. The results are just applications of the theory of one-dimensional diffusion processes and the comparison theorem of stochastic differential equations. Basic settings for Dirichlet forms and $L$-diffusion $X$ are the same as in the previous sections.

TheOrem 39. Let $M$ be an L-recurrent manifold and $u \in \operatorname{Dom}(L)_{\text {loc. Suppose that }}$ $\Gamma(u, u)$ exists and

$$
\alpha(r)=\inf _{u(x)=r} \frac{L u}{\Gamma(u, u)}(x) \quad \text { and } \quad \beta(r)=\sup _{u(x)=r} \frac{L u}{\Gamma(u, u)}(x)
$$

are well defined.

If u satisfies one of the following conditions:

(a) $\quad \int_{1}^{\infty} \exp \left(-\int_{1}^{r} \alpha(t) d t\right) d r<\infty$ and $\int_{-\infty}^{1} \exp \left(-\int_{1}^{r} \alpha(t) d t\right) d r=\infty$,

(b) $\quad \int_{1}^{\infty} \exp \left(-\int_{1}^{r} \beta(t) d t\right) d r=\infty$ and $\int_{-\infty}^{1} \exp \left(-\int_{1}^{r} \beta(t) d t\right) d r<\infty$,

then $u$ is constant.

ProOF. These conditions imply the transience of the diffusion processes (cf. $[19,24])$ defined by

$$
d \eta_{t}=d b_{t}+\alpha\left(\eta_{t}\right) d t, \quad d \xi_{t}=d b_{t}+\beta\left(\xi_{t}\right) d t
$$

Moreover, it is easy to check that (a) implies $\eta_{t} \rightarrow \infty$ as $t \rightarrow \infty$. The time change argument and the comparison argument of stochastic differential equations (cf. [18]) show that (a) implies $u\left(X_{t}\right) \rightarrow+\infty$ as $t \rightarrow \infty$ almost surely and (b) implies $u\left(X_{t}\right) \rightarrow-\infty$ as $t \rightarrow \infty$ almost surely. These contradict the recurrence of $X$.

Let $|\tau(f)|$ denote the length of the tension field $\tau(f)$ of $f$.

COROllary 40. Let $\left(M, g_{M}\right)$ be a parabolic Riemannian manifold, $\left(N, g_{N}\right)$ a Riemannian manifold and $f: M \rightarrow N$ a $C^{2}$-map. Suppose that $N$ possesses a nonnegative $C^{2}$-function $\phi$ with a continuous function $k(x)>0$ satisfying

$$
|\nabla \phi| \leq C \quad \text { and } \quad \operatorname{Hess} \phi \geq k(x) g_{N} .
$$

Set

$$
a(r)=\frac{1}{2 C^{2}} \inf _{\phi \circ f(x)=r}\left(k \circ f(x)-C \frac{|\tau(f)|}{e_{f}}(x)\right) .
$$

If

$$
\int_{1}^{\infty} \exp \left(-\int_{1}^{r} a(t) d t\right) d r<\infty
$$

then $f$ is constant. 
PROOF. Just as in [25], we have

$$
\Delta_{M}(\phi \circ f) \geq k \circ f(x) e_{f}-C|\tau(f)| \text { and }|\nabla \phi \circ f|^{2} \leq C^{2} e_{f} .
$$

We introduce an additional notation. Let $\left(M, g_{M}\right)$ and $\left(N, g_{N}\right)$ be Riemannian manifolds and $f: M \rightarrow N$ a $C^{2}$-map. Set $\tilde{g}_{N}=g_{N}-d r \otimes d r$ for $r(x)=d_{N}(o, x)$. Define

$$
\|d f\|=\|d f\|_{\left(T_{x} M, g_{M}\right) \rightarrow\left(T_{f(x)} N, \tilde{g}_{N}\right)} .
$$

COROLlARY 41. Let $N$ be a Cartan-Hadamard manifold with $\operatorname{Sect}_{N} \leq-\kappa^{2}(\kappa>0)$, $M$ a parabolic Riemannian manifold and $f: M \rightarrow N$ a harmonic map. If

$$
\int_{1}^{\infty} \exp \left(-\int_{1}^{r} \kappa \inf _{\operatorname{rof} f(x)=t}\|d f\|^{2} d t\right) d r<\infty
$$

then $f$ is constant.

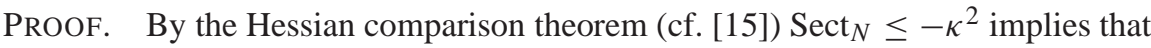

$$
\text { Hess } r \geq \kappa \frac{\cosh (\kappa r)}{\sinh (\kappa r)}\left(g_{N}-d r \otimes d r\right) \text {. }
$$

5. Transient case. We give a proof of Theorem 1 in a general situation. In this section we assume the existence of the zeroth Green function $g(x, y)$ for an $L$-diffusion on an $L$-transient manifold $M$.

THEOREM 42. Let $M$ be an L-transient manifold and $N$ a Cartan-Hadamard manifold. If $f: M \rightarrow N$ is an L-harmonic map of finite energy and if $M$ does not admit any nonconstant bounded L-harmonic functions, then $f$ is constant q.e.

Define

$$
S_{00}=\left\{\mu \text {; a smooth measure } \mid \mu(M)=1 \text { and }\left\|\int_{M} g(\cdot, y) d \mu(y)\right\|_{\infty}<\infty\right\}
$$

(cf. [13]). We first show the following.

LEMMA 43. Let $u$ be a nonnegative and finely continuous L-subharmonic function of finite L-energy on a transient manifold $M$ and $X$ an L-diffusion on $M$. Then $\left\{u\left(X_{t}\right) ; 0 \leq\right.$ $t<\zeta\}$ is a uniformly integrable submartingale under $P_{\mu}$ for any $\mu \in S_{00}$.

PROOF. The well-known Royden decomposition (cf. $[1,2,13])$ or the orthogonal decomposition of reflected Dirichlet space (cf. [4]) implies that $u$ has an $L$-harmonic function part of finite Dirichlet integral, since $M$ is transient and $u$ is of finite energy. Then $u$ has an $L$-harmonic majorant $h$. By the Burkholder inequality

$$
E_{x}\left[\sup _{0 \leq t \leq \tau_{n}} u\left(X_{t}\right)^{2}\right] \leq E_{x}\left[\sup _{0 \leq t \leq \tau_{n}} h\left(X_{t}\right)^{2}\right] \leq \text { const. } E_{x}\left[\langle h(X)\rangle_{\tau_{n}}\right]
$$

for $\tau_{n}=\inf \left\{t>0 ; X_{t} \notin G_{n}\right\}$ with an exhaustion $\left\{G_{n}\right\}$ defined as in Section 1.2. Take $\mu \in S_{00}$. Then $\int_{M} E_{x}\left[\langle h(X)\rangle_{\tau_{n}}\right] d \mu(x) \leq\left\|\int_{M} g(\cdot, y) d \mu(y)\right\|_{\infty} \mu\langle h, h\rangle(M)$. This shows $E_{\mu}\left[\sup _{0 \leq t<\zeta} u\left(X_{t}\right)^{2}\right]<\infty$. 
In the transient case we know the asymptotic behavior of $L$-harmonic maps along $L$-diffusions by Darling's martingale convergence theorem [7].

Proposition 44. Let $M$ be an L-transient manifold and $f$ an $L$-harmonic map to a Riemannian manifold $N$. Then $f\left(X_{t}\right)$ converges in $N \cup\{\infty\}$ as $t \rightarrow \zeta, P_{\mu}$-almost surely for any $\mu \in S_{00}$.

PROOF. We note only that

$$
E_{\mu}\left[[f(X), f(X)]_{\zeta}\right]=\frac{1}{2} \int_{M} \int_{M} g(y, x) d E_{f}(x) d \mu(y)<\infty .
$$

Proof OF TheOREM 42. Let $d$ be the distance of $N$ and set $r(x)=d(o, x)$ for an arbitrary reference point $o \in N$. It is well-known that if $r(x)$ is convex, then $r \circ f$ is $L$ subharmonic. Moreover, as before

$$
d \mu_{\langle r \circ f, r \circ f\rangle} \leq d E_{f}(x) .
$$

Then $r \circ f(X)$ is a uniformly integrable martingale under $P_{\mu}$. By the above proposition $\lim _{t \rightarrow \zeta} f\left(X_{t}\right)$ exists in $N, P_{\mu}$-almost surely. Since the invariant $\sigma$-field of $X$ is trivial, $\lim _{t \rightarrow \zeta} f\left(X_{t}\right)$ is a constant point, say $y_{0}$. Then $d\left(y_{o}, f(X)\right)$ is a uniformly integrable submartingale again. Hence, the submartingale property shows that $\int_{M} d\left(y_{o}, f(x)\right) d \mu(x)=0$ for arbitrary $\mu \in S_{00}$.

Appendix. In this section we present a simple statement and its proof in the Dirichlet form setting of our equivalence (i) and (ii) in Theorem 20. This was pointed out by Professor M. Takeda.

Proposition 45. Let $X$ be a strong Markov process associated with a Dirichlet space $(\mathcal{E}, \mathcal{F})$. The following conditions are equivalent:

(i) $X$ is recurrent;

(ii) for any $u \in \dot{\mathcal{F}}_{\text {loc }}$ with $\mathcal{E}(u, u)<\infty$, there exist $\left\{\phi_{n}\right\} \subset \mathcal{F} \cap C_{o}(M)$ satisfying $\phi_{n} \rightarrow 1$ as $n \rightarrow \infty$ m-almost everywhere such that

$$
\lim _{n \rightarrow \infty} \mathcal{E}\left(u, \phi_{n}\right)=0 .
$$

ProOF. (ii) $\Rightarrow$ (i) is essentially the same as in our case and we omit it. We assume that $X$ is recurrent. In this case

$$
\mathcal{F}^{\text {ref }}=\mathcal{F}_{e}
$$

holds (see [4]). This implies that $\mathcal{E}(u, u)<\infty$ means $u \in \mathcal{F}_{e}$. On the other hand, it is known (cf. [13]) that (i) is equivalent to the condition that there exits a sequence $\left\{\psi_{n}\right\} \subset \mathcal{F}$ satisfying $\lim _{n \rightarrow \infty} \psi_{n}=1 m$-almost everywhere and $\lim _{n \rightarrow \infty} \mathcal{E}\left(\psi_{n}, \psi_{n}\right)=0$. Combining these facts we see that $\mathcal{E}(u, \psi)$ for $\psi \in \mathcal{F}$ makes sense. Then we have

$$
\left|\mathcal{E}\left(u, \psi_{n}\right)\right|^{2} \leq \mathcal{E}(u, u) \mathcal{E}\left(\psi_{n}, \psi_{n}\right) \rightarrow 0 .
$$


We remark that the recurrence is equivalent to

$$
1 \in \mathcal{F}_{e} \quad \text { and } \quad \mathcal{E}(1,1)=0 .
$$

Hence,

$$
|\mathcal{E}(u, 1)|^{2} \leq \mathcal{E}(u, u) \mathcal{E}(1,1)=0 .
$$

We also note a simple argument about Theorem 12 for functions of finite Dirichlet integral.

Proposition 46. Let u be a $\delta$-L-subharmonic function of finite Dirichlet integral on $D$. Then its Riesz charge is a smooth signed measure.

Proof. By the Kunita-Watanabe inequality we see that for $\phi \in \mathcal{F} \cap C_{o}(M)$

$$
\left|\int_{D} d \mu_{\langle u, \phi\rangle}\right| \leq\left(\int_{D} d \mu\langle u, u\rangle\right)^{1 / 2}\left(\int_{D} d \mu_{\langle\phi, \phi\rangle}\right)^{1 / 2}=\operatorname{constant} \mathcal{E}(\phi, \phi)^{1 / 2} .
$$

On the other hand,

$$
-\int_{D} d \mu\langle u, \phi\rangle=\int_{D} \phi d v_{u}
$$

by definition. Thus, $v_{u}$ is of finite energy. Hence, by the general theory of Dirichlet spaces (cf. [13]), $v_{u}$ charges no sets of zero capacity.

AcKnowledgment. The author would like to thank Professor Masayoshi Takeda for allowing him to present his simple proof of a part of Theorem 20 in the Appendix and for fruitful discussions.

\section{REFERENCES}

[1] A. Ancona, Théorie du potentiel sur les graphes et les variétés, École d'été de Probabilites de Saint-Flour XVIII-1988, 1-112, Lecture Notes in Math. 1427, Springer, Berlin, 1990.

[2] A. Ancona, R. Lyons and Y. Peres, Crossing estimates and convergence of Dirichlet functions along random walk and diffusion paths, Ann. Probab. 27 (1999), 970-989.

[ 3 ] R. M. Blumenthal and R. K. Getoor, Markov processes and potential theory, Pure Appl. Math. 29, Academic Press, New York, 1968.

[ 4 ] Z. Q. ChEn, On reflected Dirichlet spaces, Probab. Theory Related Fields, 94 (1992), 135-162.

[ 5 ] S. Y. Cheng, L.-F. TAM and T. Y.-H. WAN, Harmonic maps with finite total energy, Proc. Amer. Math. Soc. 124 (1996), 275-284.

[ 6 ] M. Cranston, W. S. Kendall and P. March, The radial part of Brownian motion II, Its life and times on the cut locus, Probab. Theory Related Fields 96 (1993), 353-368.

[ 7 ] R. W. R. DARLing, Convergence of martingales on a Riemannian manifold, Publ. Res. Inst. Math. Sci. 19 (1983), 753-763.

[ 8 ] M. EMERY, Stochastic calculus in manifolds, Unversitext, Springer, Berlin, 1989.

[ 9 ] H. FederER, Geometric measure theory, Springer, Berlin, 1996.

[10] M. Fukushima, On recurrence criteria in the Dirichlet space theory, From local times to global geometry, control and physics (Coventry, 1984/85), 100-110, Pitman Res. Notes Math. 150, Longman, Harlow, 1986.

[11] M. FukUSHima, On semi-martingale characterizations of functionals of symmetric Markov processes, Electron. J. Probab. 4 (1999), 1-32. 
[12] M. Fukushima and M. OKADA, On Dirichlet forms for plurisubharmonic functions, Acta Math. 159 (1987), 171-213.

[13] M. Fukushima, Y. Oshima and M. Takeda, Dirichlet forms and symmetric Markov processes, de Gruyter Stud. Math. 19, Walter de Gruyter \& Co., Berlin, 1994.

[14] M. Fukushima, K. Sato and S. Taniguchi, On the closable parts of pre-Dirichlet forms and the fine supports of underlying measures, Osaka J. Math. 28 (1991), 517-535.

[15] R. E. GREEN and H. Wu, Function theory on manifolds which possess a pole, Lecture Notes in Math. 699, Springer, Berlin, 1979.

[16] W. K. Hayman and P. B. Kennedy, Subharmonic functions I, London Math. Soc. Monogr. 9, Academic Press, London-New York, 1976.

[17] W. K. Hayman, Subharmonic functions II, London Math. Soc. Monogr. 20, Academic Press, London-New York, 1989.

[18] N. IKEDA and S. WATANABE, Stochastic differential equations and diffusion processes, Second edition, North-Holland Math. Library, 24, North-Holland Publishing, Amsterdam, Kodansha, Tokyo, 1989.

[19] K. ITO and H. P. MCKeAn, JR., Diffusion processes and their sample paths, Second edition, Springer, Berlin, 1974.

[20] H. KAneKo, A stochastic approach to a Liouville property for plurisubharmonic functions, J. Math. Soc. Japan 41 (1989), 291-299.

[21] W. S. Kendall, Probability, convexity, and harmonic maps with small image I, Uniqueness and fine existence, Proc. London Math. Soc. (3) 61 (1990), 371-406.

[22] J. PICARD, Smoothness of harmonic maps for hypoelliptic diffusions, Ann. Probab. 28 (2000), 643-666.

[23] J. PICARD, The manifold-valued Dirichlet problem for symmetric diffusions, Potential Anal. 14 (2001), 5372.

[24] D. RevuZ and M. Yor, Continuous martingales and Brownian motion, Springer, Berlin, 1991.

[25] K. TAKegoshi, A note on divergence of $L^{p}$-integral of subharmonic functions and its applications, Proc. Amer. Math. Soc. 131 (2003), 2849-2858 (electronic).

[26] S. T. YAU, Some function-theoretic properties of complete Riemannian manifold and their applications to geometry, Indiana Univ. Math. J. 25 (1976), 659-670.

KEIO UNIVERSITY

4-1-1, НiYOSHI, YOKOHАMA 223-8521

JAPAN

E-mail address: atsuji@math.hc.keio.ac.jp 